\title{
The Basis of Atherosclerotic Guidelines-Time for a Change?
}

\author{
David S. Schade, R. Philip Eaton \\ Division of Endocrinology, Department of Internal Medicine, University of NM Health Sciences Center, Albuquerque, NM, USA \\ Email: dschade@salud.unm.edu
}

How to cite this paper: Schade, D.S. and Eaton, R.P. (2018) The Basis of Atherosclerotic Guidelines-Time for a Change? World Journal of Cardiovascular Diseases, 8, 337-352.

https://doi.org/10.4236/wjcd.2018.87033

Received: June 14, 2018

Accepted: July 22, 2018

Published: July 25, 2018

Copyright $\odot 2018$ by authors and Scientific Research Publishing Inc. This work is licensed under the Creative Commons Attribution International License (CC BY 4.0).

http://creativecommons.org/licenses/by/4.0/

(c) (i) Open Access

\begin{abstract}
Major advances have occurred within the last decade in the understanding of the pathogenesis of coronary artery disease. Not only are the underlying mechanisms now clearly defined, but effective medical therapies are available at low cost and minimal side effects. In spite of these advances, cardiovascular events are still the leading cause of death in the United States and the Western world. Analysis of the many factors involved in the delivery of appropriate cardiovascular care strongly suggests that the primary reason is the overly restrictive guidelines published by medical societies. This article proposes a much broader basis for constructing atherosclerosis clinical guidelines, namely the known pathophysiology of atherosclerosis. If pathophysiology forms the basis of atherosclerotic treatment recommendations, then a risk/benefit analysis can be used to determine appropriate preventive therapy for any specific individual. The result will be that many additional individuals will be eligible for preventive treatment of atherosclerosis, and the saving of many lives at minimal cost will result.
\end{abstract}

\section{Keywords}

Randomized Controlled Trials, Pathophysiology, Clinical Practice Guidelines, Internal Validity, External Validity, Target-Based Therapy

\section{Introduction}

The choice of pathophysiology-based clinical guidelines versus randomized clinical trials (RCTs) based clinical guidelines has critical bearing upon the increasing prevalence of atherosclerotic heart disease in the United States [1]. Fundamentally, pathophysiology-based clinical guidelines support risk/benefit considerations for patient specific clinical management by physicians. In contrast, randomized clinical trials based clinical guidelines support the mean pop- 
ulation response to a drug or lifestyle intervention and therefore have no proven outcome for any individual patient to achieve his/her optimal end point response. The result is that many of the individuals with significant undiagnosed atherosclerosis are not being appropriately treated and suffer cardiovascular events.

It may be surprising that the majority of professional organizations that issue cardiovascular guidelines base their recommendations primarily on the results of randomized controlled trials [2]. Rather than considering the risk/benefit ratio of a recommendation for a patient from the typical comorbid diverse clinical population, RCTs report a "proven" fact for a highly selected non-representative group within a $95 \%$ confidence interval. The result is that many individuals who would benefit from a relatively benign, inexpensive therapy are prevented from receiving treatment. Because they do not qualify for the RCT study group, they experience a major cardiovascular event.

The difference between randomized control based guidelines and pathophysiological based guidelines is illustrated in Figure 1. Both approaches begin with the same heterogeneous population. However, to ensure internal validity by studying a relatively homogeneous population, the randomized control based guideline approach follows a stepwise exclusion (reduction in the size) of the population to be tested. In contrast, the pathophysiological based guideline is not restricted to a homogenous population and therefore does not restrict the number of individuals to be included in the assessment. This difference is significant because there are many individuals with atherosclerosis that need to be treated with medication but are excluded because their personal characteristics were not included in available clinical trials. These individuals usually include the elderly, the young, and people with multiple medical issues.

Atherosclerosis belongs to a collection of diseases in which the pathophysiology involving abnormalities in the blood must be corrected to achieve a successful clinical outcome. The list of such pathophysiology-based diseases is long, and ranges from gout, in which blood uric acid levels must be normalized to prevent the inflammation of joints, to AIDS in which the virus in the blood must be suppressed or eliminated to prevent clinical complications. Table 1 contains a partial list in which guidelines identify the therapeutic target concentration of the offending agent for correction to successfully eliminate the clinically expressed disease. In this group of diseases, treating for a population mean outcome based on randomized controlled trial guidelines may fail to cure the disease in those patients who fall outside of the mean RCT population reduction in concentration of the causative agent in the blood.

From a historical perspective, each of these diseases in Table 1 was treated with limited success with many different pharmacological and medical approaches for decades prior to identifying the single abnormality within the blood that was the essential pathophysiological abnormality. Atherosclerosis treatment guidelines evolved initially toward improving clinical cardiac outcomes as the 

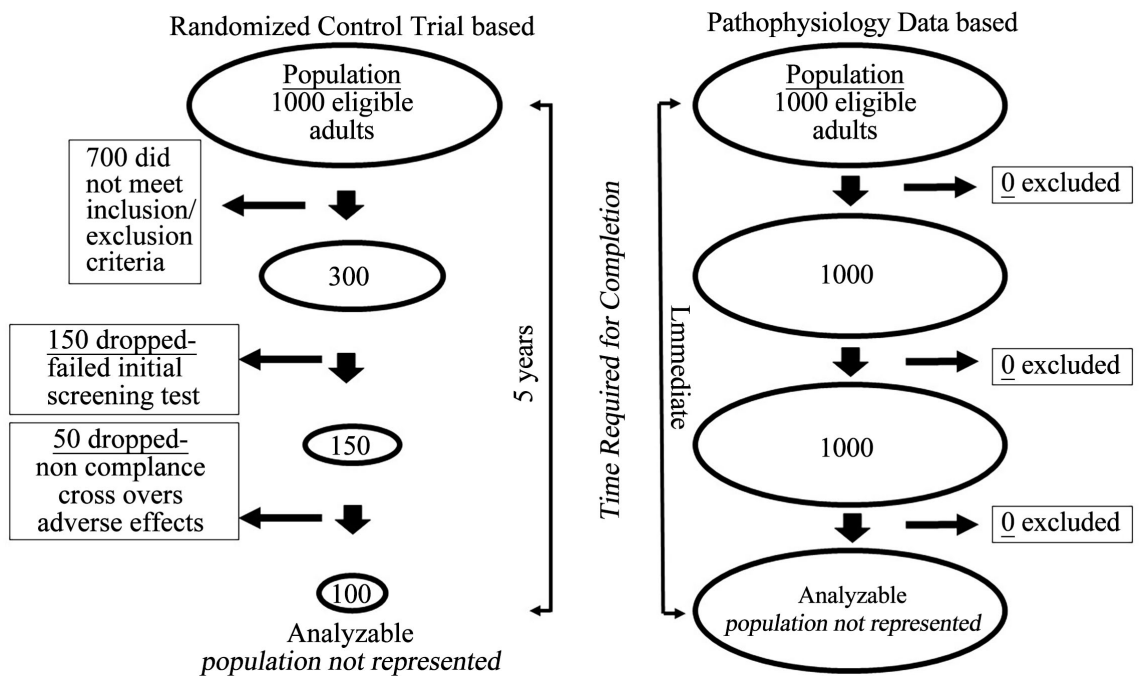

Figure 1. A comparison between guidelines that are randomized controlled based (RCT) and guidelines that are based on pathophysiology. In an attempt to study a homogeneous population, there are many exclusions for RCT based guidelines (which reduce the cost and duration and also increases the statistical power of the study). Unfortunately, this approach leads to a study of non-representative populations (poor external validity). In contrast, pathophysiologically based guidelines apply to all of the population and permit immediate analysis of all the currently available data.

Table 1. Examples of blood treatment targets causing disease.

\begin{tabular}{cc}
\hline Substances & Resulting Disease \\
\hline Uric acid & Gout \\
Iron & Hemochromatosis \\
Copper & Wilson's disease \\
Hormones & \\
Thyroid & Thyrotoxicosis \\
Growth Hormone & Acromegaly \\
Cortisol & Cushing's disease \\
Infectious agents & \\
Hepatitis C virus & Hepatitis C \\
Chickenpox virus & Shingles \\
\hline
\end{tabular}

role of an underlying offending agent has taken over 40 years to evolve into pathophysiology-based recognition. For example, bypass surgery of coronary arteries followed later by catheter placed vessel stents quickly demonstrated dramatic resolution of acute coronary occlusion. However, it has become clear that coronary artery stents do not correct the pathology of the underlying vessel and future atherosclerotic events are not prevented. Thus, coronary artery based disease has remained the number one cause of death in the United States [3].

Based upon the accumulation of abundant pathophysiologic data today, it is 
the hypothesis of this review to propose that at least two agents, circulating LDL cholesterol (LDLc) and inflammation as measured by hsCRP, represent a treatment target for pathophysiology-based clinical guidelines in patients bearing a significant atherosclerotic burden responsible for coronary heart disease.

Atherosclerotic burden is identified in asymptomatic patients with calcium scanning [Figure 2], and in symptomatic patients by history of heart attack with or without stent or bypass intervention. Based upon pathophysiology in these patients, physicians should consider medication titration to achieve the targeted blood LDLc concentration reduction levels to maximally prevent clinical cardiac events and reverse atherosclerosis.

\section{Background}

The pathways to today's challenge of treatment of coronary atherosclerosis began before the turn of the century with the pathologic description of fatty atheroma within the heart vessels and major arteries. It required a hundred years before the clinical recognition of heart attacks as the number one cause of death in the United States led to the identification of effective interventions. It became understood that the unstable, cholesterol filled, inflammatory atherosclerotic plaque is the culprit leading to coronary artery thrombosis and death [4]. Rupture of the plaque's fibrous cap and exudation of the thrombogenic contents results in arterial coagulation and coronary occlusion [5] [6].

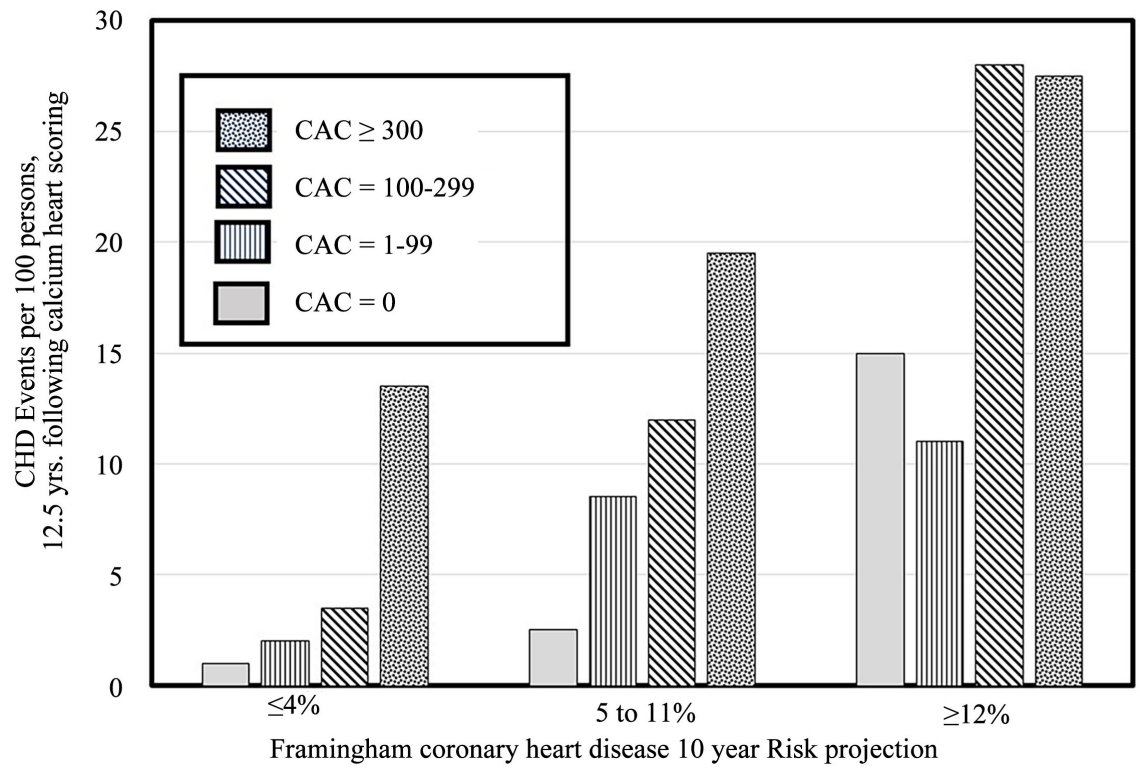

Figure 2. The value of the coronary calcium heart scan in predicting CHD events. A progressive increase in $\mathrm{CHD}$ at 12.5 years was observed with increasing coronary calcium at baseline. The figure also suggests that the CAC scan should be repeated every 5 years to allow for identification of newly positive scans and the adjustment of risk assessment. This suggestion is based on the observation that CHD events were observed in the high Framingham risk category $(>12 \%)$ when the original score was zero a decade before the event. Data modified from ref [32]. 
For the next fifty years, two pathways of a solution were followed in almost parallel trajectories. One was directed at surgical removal of a damaged site in a coronary vessel, and one was directed at the biochemical role of "fat/cholesterol" as recognized chemically in cutaneous xanthoma in families with early presentation of heart attacks. Within a decade, the effect of emergent by-pass surgery in saving patients with acute vessel occlusion was reported and replicated [7]. Within twenty years, this surgical success was followed with similar life-saving outcome utilizing catheter-based vessel dilating stents. While this became the standard of acute management, a reduction in the demonstrated role of heart attacks as the number one cause of death was not observed. During the same 40 years, biochemical investigation achieved two critical advances. The first was the ability to identify and clinically measure various species of cholesterol lipoproteins in the blood. Specifically, the presence of LDL cholesterol in abnormally elevated lipid levels in children and their parents with a history of early death by heart attacks suggested an inherited molecular disease. This possibility historically suggested searching for a biochemical abnormality as a basis for the underlying atheroma. With advances in molecular genetics emerging, the demonstration of hepatic LDL cholesterol defective receptors was confirmed, and quickly biochemical intervention with inhibitors of cholesterol synthesis were pursued.

The second clinical advance was the demonstration that HMG-CoA reductase inhibition of cholesterol synthesis with "statins" reduced the test population incidence of heart attacks in randomized clinical trials of qualifying high-risk patients. These trials of a single dosage of drug were pursued for the next ten years with statistical and meta-analysis confirmation to demonstrate equivalent success to that achieved with vessel surgery [8]. The results were not related to individual patient changes in LDLc, but only to the population mean LDLc level. Heart attacks were indeed reduced, and guidelines issued recommending a single daily dosage of a statin as a therapeutic intervention [9]. Without any attempt to achieve a uniform lowering of LDLc in the statin trials, no expectations for target reductions in individual patients were made [10]. Unfortunately, death from cardiovascular disease remained the number one cause of mortality in the United States.

\section{Increasing Importance of Pathophysiology}

While both vascular surgery and single-dose statin therapeutic interventions continued, a third pathway of clinical investigation based upon "pathophysiology" was actively being pursued by many scientists around the world. Historically, this approach had been the most common approach to determining therapeutic target concentrations of disease agents such as serum uric acid, lead, BUN, thyroxin, cortisone, etc. Pathophysiology includes all scientific studies that describe a disease process either directly or indirectly. These studies include (but are not limited to) observational, in vitro, cross sectional, animal, database, case reports, and randomized controlled trials (Figure 3). The great strength of 


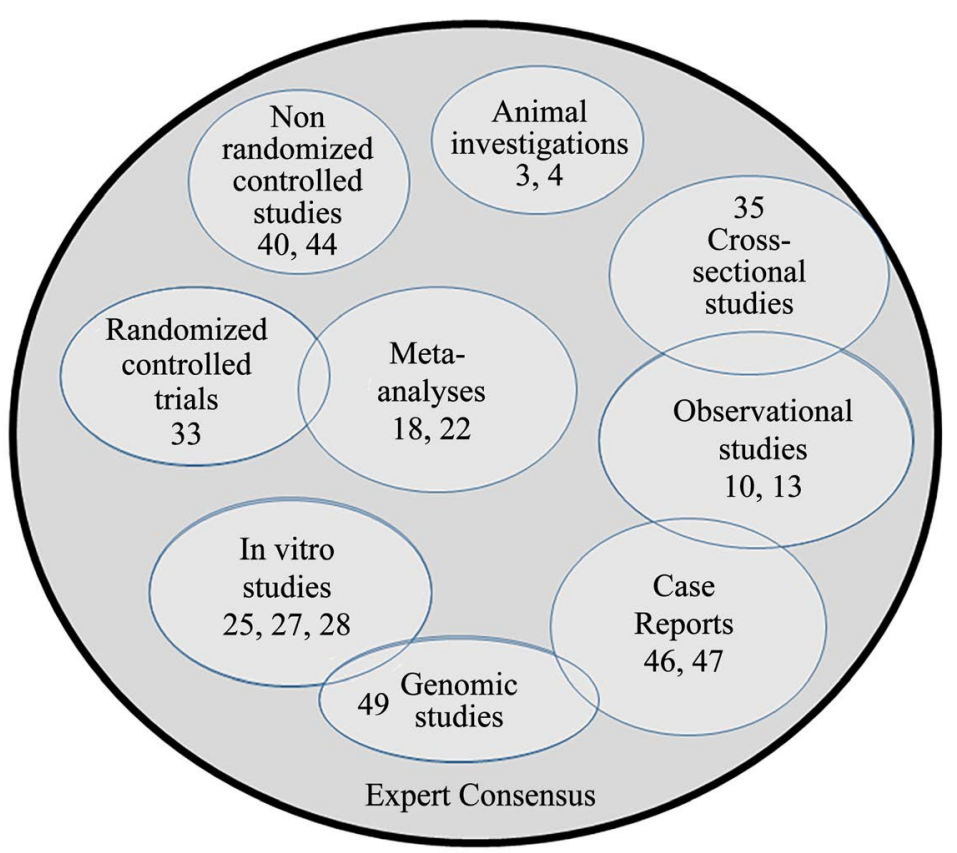

Figure 3. A scaled Venn diagram demonstrating the components contributing to the pathophysiology of atherosclerosis. The size of the circles can be used to provide the relative weight that each component was given toward a treatment recommendation. Overlapping circles indicate that some studies fall into more than one category (not all overlaps are shown for reasons of simplicity). The overall "expert consensus" circle includes the consideration of all the available pathophysiological data pertinent to a specific disease's treatment and forms the basis of the recommendations. As a hypothetical example, the numbers (inside the circles) indicate the specific references that are cited in the professional organization's guidelines that have the most influence in constructing their recommendations. The inclusion of this type of diagram increases the communication between the reader and the authors of the clinical practice guidelines by permitting the reader to understand the reasons for the recommendations.

pathophysiology is that, a priori, it is continuously strengthened by new discoveries and insights into a disease that might occur.

Treatment practice based upon pathophysiology guidelines can utilize a risk/benefit analysis typical of everyday clinical practice, so that potential unknown risks can be minimized. This allows safe, aggressive therapy to be applied to the individual patient with a potential life threatening disease. Finally, by preventing/reversing atherosclerosis, the patient can achieve favorable outcomes that are both cost effective to the individual and to the U.S. health care system [11].

The human genetic data in familial hypercholesterolemia have clearly demonstrated that members of a family with elevated levels of LDLc have heart attacks prematurely. In contrast, relatives with LDL cholesterol concentrations more typical of the general population did not seem to have premature heart attacks. Close examination of the statin randomized control trials revealed a similar possibility, even though no target level of LDLc was utilized [12]. When one did a comparison of the many trials with differing statin agents and/or using differing 
statin dosages, a statistical relationship between the degree of LDL reduction using population means, and the resulting reduced heart attack events could be recognized [13]. This raised the question of whether or not individual management of LDLc lowering to a specific blood level might be considered. Pathophysiology based practice has been the traditional knowledge-based process to pursue this possible role of a concentration-based agent causing tissue disease in the individual patients within a primary care practice.

Recognizing that subhuman primates rarely develop atherosclerosis, other animal models of atherosclerosis were developed [14]. Discovery of the Watanabe atherosclerotic prone rabbit provided a unique resource to study the atherosclerotic process [15]. Identification of the hepatic LDL receptor by Brown and Goldstein and proof of its role in homozygous hypercholesterolemia and reversal by liver transplant provided the scientific basis for direct hepatic inhibition of cholesterol synthesis [16] [17]. Meta-analysis of 90,056 subjects from 14 randomized clinical trials of statins demonstrated the ability to safely reduce hepatic cholesterol synthesis with this class of medications [18]. More recent genomic studies of individuals with either inherited increased or decreased atherosclerotic events related to LDL cholesterol concentration has further elucidated the pathogenesis of atherosclerosis depending upon LDLc levels, and the development of new approaches to the treatment of this disease [19]. Furthermore, analysis of genomic traits that result in a reduced LDL concentration from early childhood indicates that this trait is associated with a substantially greater reduction in cardiovascular events than the current practice of lowering LDL later in life [20] [21].

Finally, it has been recently reported that a clonal hematopoiesis of indeterminate potential (CHIP) mutation in adults over the age of $70 \mathrm{yrs}$. is significantly associated with increased heart attack and strokes [22]. From a pathophysiologic perspective, these authors relate this observation to the increased atherosclerotic inflammation in genetic mice models of familial hypercholesterolemia, but not in normal cholesterol control mice. The interaction of circulating cholesterol levels and tissue inflammation is an associated area of important advances that further support the pathophysiologic approach to evolving therapy for heart attacks. The CHIP genetic inflammatory link is consistent with the direct quantification of calcium presence as an inflammatory component of coronary atherosclerotic plaques in man. This virtual biopsy confirmation of the amount of atherosclerotic burden in coronary vessels by coronary artery calcium scan has proved to be statistically linked with the risk of cardiac events during 5 to 15 years of follow-up [18] [23]. The calcium score is independent of clinical scoring based upon clinical risks including hypertension, diabetes, age, etc., or can be improved with the standard risk-based prediction such as the Framingham guidelines [24]. Including direct quantification of coronary atherosclerotic burden identifies those asymptomatic patients in whom targeted LDL reduction is necessary to reverse the atherosclerosis. 
Of critical importance is that atherosclerosis is a reversible disease [25]. Atherosclerotic regression following LDL cholesterol reduction was first reported almost two decades ago [26], and more recent studies demonstrated that it was reversible within one year in subjects with known cardiovascular disease [11] [27] [28] [29]. Of most importance, investigation of the effect of targeted reduction in LDL cholesterol was addressed. Studying 1139 individuals with and without diabetes, reducing the circulating LDL below $70 \mathrm{mg} / \mathrm{dl}$ reversed atherosclerosis [30]. It was then observed that all LDL-lowering therapies that increase the hepatic LDL receptor while reducing LDL cholesterol concentration were effective in reducing cardiovascular events (including lifestyle changes, statins, ezetimibe, ileal bypass, and PCSK9 inhibitors); whereas therapies that do not increase the hepatic LDL receptor concentration don't reduce cardiovascular events (e.g., niacin) [31]. In addition, when cardiovascular clinical trials employing statins examined the relationship of LDL lowering with the reduction of cardiovascular events, it was consistently observed that the lower the LDL concentration, the fewer the events [32]. This concept has been further extended by a recent trial of the PCSK9 inhibitor evolocumab that demonstrated the benefits of even further reduction of circulating LDL cholesterol from $70 \mathrm{mg} / \mathrm{dl}$ to 30 $\mathrm{mg} / \mathrm{dl}$ in high-risk individuals [33]. Finally, understanding the pathophysiology of atherosclerosis provides reassurance that low LDL cholesterol levels are safe, at least down to $30 \mathrm{mg} / \mathrm{dl}$ [34]. Many humans are born with LDL levels in the 50 $\mathrm{mg} / \mathrm{dl}$ range [35]. Individuals with a genetic deficiency of PCSK9 may have lifetime circulating LDLc levels as low as $14 \mathrm{mg} / \mathrm{dl}$ without adverse effects [33].

\section{Effect of Pathophysiology Based Target Guidelines on Heart Disease}

Atherosclerosis is the number one cause of mortality in the United States, more than all cancers combined [36]. Although the acute mortality has been slowly decreasing secondary to improved emergency cardiovascular interventions, the actual prevalence of the disease continues to increase [1]. Approximately 600,000 individuals in the United States suffer a cardiovascular event (myocardial infarction or stroke) each year. Half of these patients die before reaching the hospital, and according the American Heart Association, a recent estimate of the cost of atherosclerotic cardiovascular disease is 207 billion dollars per year [2]. Because atherosclerosis is a silent killer, at least $50 \%$ of these patients were asymptomatic and unaware that they were at risk.

Guidelines based upon pathophysiology begin by recognizing that atherosclerosis is a chronic disease that begins in childhood. Knowing that $5 \%$ of our population has familial heterozygous hypercholesterolemia, consideration could address those at the interface between teenagers and young adults. This is the current practice in pediatric management of young adult with homozygous familial hypercholesterolemia [37]. When atherosclerotic burden is identified noninvasively within the heart by coronary artery calcium scanning in at-risk members 
of such families, then consideration of targeted medical lowering of their LDL cholesterol to $<50 \mathrm{mg} / \mathrm{dl}$ is appropriate. The next category is men and women who have experienced a heart attack, stroke, by-pass or stent in whom these events confirm the presence of atherosclerosis. These pathophysiological based recommendations would identify targeted medical lowering of their LDL cholesterol to $<50 \mathrm{mg} / \mathrm{dl}$. Finally, any man or women who is considered "at-risk" for coronary disease by evaluations such as the Framingham risk calculations, and who has a positive atherosclerotic burden by cardiac calcium scan, would qualify for reducing the LDL cholesterol to $<50 \mathrm{mg} / \mathrm{dl}$.

The medications currently available are appropriate to achieve a target blood LDLc concentration of $<50 \mathrm{mg} / \mathrm{dl}$ by following the patient's response with titration to achieve this goal. Based on the known side effects of lifestyle change, statins, ezetimibe, and low dose aspirin (all of which have been in use for more than 20 years), the benefit of the pathophysiological approach greatly outweighs any therapy related risks [38]. Since all of the cited therapies are now generic, the prevention of cardiovascular disease is very cost effective, with medication costs of less than one dollar per day. Furthermore, because of the anticipated cost saving from the prevention of emergency acute coronary care, many medical insurance companies are now providing statins and ezetimibe at no or minimal charge to their enrollees.

Figure 4 illustrates the estimated difference between the numbers of individuals in the Unites States that would be recommended for therapy to prevent atherosclerosis based upon the pathophysiologic target LDL cholesterol level $<50$ $\mathrm{mg} / \mathrm{dl}$ compared with therapy reflecting guidelines based upon randomized controlled trials. Randomized controlled trials limit eligible individuals to the age groups that have been studied between ages 40 - $70 \mathrm{yrs}$, and exclude adults with no cardiac risk factors or low Framingham scores. They do not establish the presence or absence of an atherosclerotic burden so that a subject may be misdiagnosed in both categories of having or not having confirmed vascular disease [39] [40] [41]. Fundamentally, pathophysiological based guidelines would include an additional 30,000,000 adults and all patients would have a known burden of coronary atherosclerosis by non-invasive cardiac calcium scanning. Since this is a "chronic" disease beginning in childhood, the simple and inexpensive cardiac calcium scan in both asymptomatic as well as high-risk men and women may be the only fail-proof way of establishing the presence of an atherosclerotic burden, the basic pathophysiologic evidence of vessel disease. Moreover, it has also been reported that left untreated, all levels of coronary artery calcium scoring may progress over time, including those with an initial calcium score of zero. This is a chronic disease and strongly influenced by any acquired inflammatory disease occurring at any time throughout a lifetime, such as influenza [42]. Considering a physician's risk/benefit approach to an individual patient, follow-up calcium scans at 5 year intervals can be considered highly cost-effective, and particularly so in the presence of other known cardiovascular risk factors. 




Figure 4. A comparison of the size of the estimated population that would qualify for treatment (lifestyle change, medication) to prevent atherosclerotic heart disease. Almost twice as many individuals would be recommended for interventional therapy when pathophysiology is used as the basis of treatment recommendations. Since RCT based treatment relies on a published risk score, many patients will be treated that do not have atherosclerotic disease. In contrast, pathophysiology based treatment only recommends treatment for individuals with a positive coronary artery calcium score, therefore, only patients with atherosclerotic disease are treated.

Not surprisingly, there are major differences between all clinical practice guidelines. The American College of Cardiology and the American Heart Association most recent guidelines of 2013 did not recommend LDL cholesterol target therapy. However, in 2017 the American Association of Clinical Endocrinologists and American College of Endocrinology suggested LDL cholesterol treatment goals of $<55 \mathrm{mg} / \mathrm{dl}$ for patients with "Extreme Risk", $<70$ with "Very High risk", $<100$ with "High Risk", and $<130$ with "Low Risk" [43]. These differences result in confusion for physicians attempting to apply them to their patients because the term "risk" is defined differently by different on-line calculators [44] [45] [46] [47]. In addition, the weaknesses of randomized controlled trials have been frequently pointed out by several authors [48] [49]. Table 2 lists these weaknesses and compares these parameters to those of pathophysiology based guidelines. There are major differences between RCT based guidelines and pathophysiology-based guidelines as shown in Table 2. The benefits of pathophysiology-based guidelines are based on the fact that they include not only RCT data but also include all other types of scientific evidence. They can also be applied immediately to the general at risk population whereas RCT based guidelines require years for studies to be completed at great cost to the population. Their narrow focus precludes general applicability to many individuals in the 
Table 2. Comparison of RCT-based and pathophysiology-based guidelines.

\begin{tabular}{|c|c|c|c|}
\hline & Parameter & $\begin{array}{l}\text { RCT based } \\
\text { guidelines }\end{array}$ & $\begin{array}{l}\text { Pathophysiology based } \\
\text { guidelines }\end{array}$ \\
\hline 1 & External validity & Very poor & Very good \\
\hline 2 & Internal validity & Very good & Good \\
\hline 3 & $\begin{array}{l}\text { Agreement among } \\
\text { Societies }\end{array}$ & Very poor & Not tested \\
\hline 4 & $\begin{array}{c}\text { Influence on } \\
\text { Primary Care givers }\end{array}$ & Minimal & $\begin{array}{c}\text { Not tested, but expectation } \\
\text { high }\end{array}$ \\
\hline 5 & $\begin{array}{l}\text { Applicability to large \# of } \\
\text { patients }\end{array}$ & Moderate & High \\
\hline 6 & Scientific basis & Narrow & Very broad \\
\hline 7 & Types of studies considered & Primarily RCTs & All scientific studies \\
\hline 8 & $\begin{array}{l}\text { Difficulty of constructing } \\
\text { guidelines }\end{array}$ & Moderate & High \\
\hline 9 & Cost of medical treatment & Minimal & Minimal \\
\hline 10 & Cost of surgical treatment & Very high & Very high \\
\hline
\end{tabular}

population (i.e., poor external validity). Because atherosclerosis is the number one cause of death in the Western world, the risk/benefit analysis strongly favors pathophysiology-based guidelines. The pathophysiological significance of documenting vessel atherosclerosis burden by calcium scan and reducing the LDL cholesterol to $<50 \mathrm{mg} / \mathrm{dl}$, significantly differentiates these two approaches to therapy guidelines for this chronic disease [Figure 2].

\section{Conclusion}

This position paper poses the hypothesis that pathophysiologic data establishing a target lowering of LDL cholesterol and hsCRP in all asymptomatic patients with coronary atherosclerosis and symptomatic patients with a clinical history of cardiovascular disease will reduce the number of patients dying of cardiovascular disease. It acknowledges that randomized controlled trials are often the preferred experimental design for answering therapeutic questions in very specific populations, but they exclude the realty of comorbid diseases that characterize the general clinic population. We are not the first authors to raise issues concerning clinical practice guidelines, but we are the first to offer a feasible alternative approach. Pathophysiology provides the professional organization with the ability to factor in non-randomized controlled studies and alter their therapeutic recommendations in a timely fashion. In many circumstances, it is also cost effective, preventing the high cost of invasive long-term cardiovascular medical care [50]. Most importantly, it permits recommendations that are directly applicable to the general clinic population, not just the carefully selected volunteers enrolled in randomized controlled trials. The beneficiaries of utilizing pathophysiology will be both the physicians who rely on guidelines and their patients who 
will receive the most current therapy based upon all the available experimental data to achieve a target level of reduced LDL and hsCRP concentration.

\section{Acknowledgements}

D.S.S. and R.P.E. appreciate the contributions of Deirdre Hill, PhD, Division of Epidemiology, University of New Mexico Health Sciences, for statistical suggestions to our manuscript.

\section{References}

[1] Fuster, V. (2009) The CVD Paradox: Mortality vs. Prevalence. Nature Reviews Cardiology, 6, 669. https://doi.org/10.1038/nrcardio.2009.187

[2] Khanji, M.Y., Bicalho, V.S., van Waardhuizen, C.N., Ferket, B.S., Petersen, S.E. and Myriam Hunink, M. (2016) Cardiovascular Risk Assessment: A Systematic Review of Guidelines. Annals of Internal Medicine, 165, 713-722.

[3] Mozaffarian, D., Benjamin, E.J., Go, A.S., Arnett, D.K., Blaha, M.J., Cushman, M. Das, S.R. and de Ferranti, S. (2016) American Heart Association Statistics Committee; Stroke Statistics Subcommittee. Heart Disease and Stroke Statistics-2016 Update: A Report from the American Heart Association. Circulation, 133, e38-360. https://doi.org/10.1161/CIR.0000000000000350

[4] Libby, P. (2005) The Forgotten Majority: Unfinished Business in Cardiovascular Risk Reduction. Journal of the American College of Cardiology, 46, 1225-1228. https://doi.org/10.1016/j.jacc.2005.07.006

[5] Kern, M.J. and Meier, B. (2001) Evaluation of the Culprit Plaque and the Physiological Significance of Coronary Atherosclerotic Narrowings. Circulation, 103, 3142-3149. https://doi.org/10.1161/01.CIR.103.25.3142

[6] Arbab-Zadeh, A., Nakanano, M., Virmani, R. and Fuster, V. (2012) Acute Coronary Events. Circulation, 125, 1147-1156.

https://doi.org/10.1161/CIRCULATIONAHA.111.047431

[7] Chaitman,j B.R., Fisher, L.D., Bourassa, M.G., Davis, K., Rogers, W.J., Maynard. C., et al. (1981) Effect of Coronary Bypass Surgery on Survival Patterns in Subsets of Patients with Left Main Coronary Artery Disease: Report of the Collaborative Study in Coronary Artery Surgery (CASS). American Journal of Cardiology, 48, 765-777 https://doi.org/10.1016/0002-9149(81)90156-9

[8] Pitt, B., Waters, D., Brown, W.V., van Boven, A.J., Schwartz, L., Title, L.M., et al. (1999) Aggressive Lipid-Lowering Therapy Compared with Angioplasty in Stable Coronary Artery Disease. Atorvastatin versus Revascularization Treatment Investigators. The New England Journal of Medicine, 341, 70-76. https://doi.org/10.1056/NEJM199907083410202

[9] Stone, N., Robinson, J.G., Lichtenstein, A.H., Bairey Merz, C.N., Blum, C.B., Eckel, R.H., et al. (2014) 2013 ACC/AHA Guideline on the Treatment of Blood Cholesterol to Reduce Atherosclerotic Cardiovascular Risk in Adults: A Report of the American College of Cardiology/American Heart Association Task Force on Practice Guidelines. Journal of the American College of Cardiology, 63, 2889-2934. https://doi.org/10.1016/j.jacc.2013.11.002

[10] Steenhuysen, J. and Berkrot, B. (2014) New Data Fuels Doctors' Demands to Rewrite U.S. Heart Guidelines. Reuters. http://in.reuters.com/article/2014/11/21/us-health-heart-cholesterol-idUSKCN0J50 $\underline{E} 320141121$ 
[11] Nicholls, S.J., Sipahi, I. and Tuzcu, E.M. (2006) Assessment of Progression and Regression of Coronary Atherosclerosis by Intravascular Ultrasound. A New Paradigm Shift? Revista Española de Cardiología, 59, 57-66. https://doi.org/10.1157/13083650

[12] Nicholls, S.J., Ballantyne, C.M., Barter, P.J., Chapman, J., Erbel, R.M., Libby, P., et al. (2011) Effect of Two Intensive Statin Regimens on Progression of Coronary Disease. The New England Journal of Medicine, 365, 2078-2087. https://doi.org/10.1056/NEJMoa1110874

[13] Baigent, C., Keech, A., Kearney, P.M., Blackwell, L., Buck, G., Pollicino, C., et al. (2005) Efficacy and Safety of Cholesterol-Lowering Treatment: Prospective Meta-Analysis of Data from 90,056 Participants in 14 Randomised Trials of Statins. Lancet, 366, 1267-1278. https://doi.org/10.1016/S0140-6736(05)67394-1

[14] Zaragoza, C., Gomez-Guerrero, C., Martin-Ventura, J.L., Blanco-Colio, L., Lavin, B., Mallavia, B., et al. (2011) Animal Models of Cardiovascular Diseases. Journal of Biomedicine and Biotechnology, 2011, Article ID: 497841. https://doi.org/10.1155/2011/497841

[15] Aliev, G. and Burnstock, G. (1998) Watanabe Rabbits with Heritable Hypercholesterolaemia: A Model of Atherosclerosis. Histology and Histopathology, 3, 797-817.

[16] Goldstein, J.L. and Brown, M.S. (2009) History of Discovery: The LDL Receptor. Arteriosclerosis, Thrombosis, and Vascular Biology, 29, 431-438. https://doi.org/10.1161/ATVBAHA.108.179564

[17] Bilheimer, D.W., Goldstein, J.L., Grundy, S.M., Starzl, T.E. and Brown, M.S. (1984) Liver Transplantation to Provide Low-Density-Lipoprotein Receptors and Lower Plasma Cholesterol in a Child with Homozygous Familial Hypercholesterolemia. The New England Journal of Medicine, 311, 1658-1664. https://doi.org/10.1056/NEJM198412273112603

[18] Budoff, M.J., Young, R., Burke, G., Carr, J.J., Detrano, R.C., et al. (2018) Ten-Year Association of Coronary Aratery Calcium with Atherosclerotic Cardiovascular Disease (ASCVD) Events: The Multi-Ethnic Study of Atherosclerosis (MESA). European Heart Journal, 39, 2401-2408.

[19] Nioi, P., Sigurdsson, A., Thorleifsson, G., Helgason, H., Agustsdottir, A.B., Norddahl, G.L., et al. (2016) Variant ASGR1 Associated with a Reduced Risk of Coronary Artery Disease. The New England Journal of Medicine, 374, 2131-2134. https://doi.org/10.1056/NEJMoa1508419

[20] Ference, B.A., Yoo, W., Alesh, I., Mahajan, N., Mirowska, K.K., Mewada, A., et al. (2012) Effect of Long-Term Exposure to Lower Low-Density Lipoprotein Cholesterol Beginning Early in Life on the Risk of Coronary Heart Disease: A Mendelian Randomization Analysis. Journal of the American College of Cardiology, 60, 2631-2639. https://doi.org/10.1016/j.jacc.2012.09.017

[21] Ference, B.A., et al. (2015) Effect of Naturally Random Allocation to Lower Low-Density Lipoprotein Cholesterol on the Risk of Coronary Heart Disease Mediated by Polymorphisms in NPC1L1, HMGCR, or both: A $2 \times 2$ Factorial Mendelian Randomization Study. Journal of the American College of Cardiology, 65, 1552-1561. https://doi.org/10.1016/j.jacc.2015.02.020

[22] Jaiswal, S., Natarajan, P., Silver, A.J., Gibson, C.J., Bick, A.G., Shvartz, E., et al. (2017) Clonal Hematopoiesis and Risk of Atherosclerotic Cardiovascular Disease. The New England Journal of Medicine, 377, 111-121. https://doi.org/10.1056/NEJMoa1701719

[23] Carr, J.J., Jacobs, D.R., Terry, J.G., Shay, C.M., Sidney, S., Liu, K., et al. (2016) Association of Coronary Artery Calcium in Adults Aged 32 to 46 Years with Incident 
Coronary Heart Disease and Death. JAMA Cardiology, 2, 391-399.

[24] McClelland, R.L., Jorgensen, N.W., Budoff, M., Blaha, M.J., Post, W.S., Kronmal, R.A., et al. (2015) 10-Year Coronary Heart Disease Risk Prediction Using Coronary Artery Calcium and Traditional Risk Factors Derivation in the MESA (Multi-Ethnic Study of Atherosclerosis) with Validation in the HNR (Heinz Nixdorf Recall) Study and the DHS (Dallas Heart Study). Journal of the American College of Cardiology, 66, 1643-1653. https://doi.org/10.1016/j.jacc.2015.08.035

[25] Keraliya, A. and Blankstein, R. (2017) Regression of Coronary Atherosclerosis with Medical Therapy. The New England Journal of Medicine, 376, 1370. https://doi.org/10.1056/NEJMicm1609054

[26] Brown, G., Albers, J.J., Fisher, L.D., Schaefer, S.M., Lin, J.T., Kaplan, C., et al. (1990) Regression of Coronary Artery Disease as a Result of Intensive Lipid-Lowering Therapy in Men with High Levels of Apolipoprotein B. The New England Journal of Medicine, 323, 1289-1298. https://doi.org/10.1056/NEJM199011083231901

[27] Jensen, L.O., Thayssen, P., Pedersen, K.E., Stender, S. and Haghfelt, T. (2004) Regression of Coronary Atherosclerosis by Simvastatin: A Serial Intravascular Ultrasound Study. Circulation, 110, 265-270.

https://doi.org/10.1161/01.CIR.0000135215.75876.41

[28] Nicholls, S.J., Tuzcu, E.M., Sipahi, I., Grasso, A.W., Schoenhagen, P., Hu, T., Wolski, K., et al. (2007) Statins, High-Density Lipoprotein Cholesterol, and Regression of Coronary Atherosclerosis. JAMA, 297, 499-508. https://doi.org/10.1001/jama.297.5.499

[29] Nissen, S.E., Nicholls, S.J., Wolski, K., Nesto, R., Kupfer, S., Perez, A., et al. (2008) Comparison of Pioglitazone vs. Glimepiride on Progression of Coronary Atherosclerosis in Patients with Type 2 Diabetes: The PERISCOPE Randomized Controlled Trial. JAMA, 299, 1561-1573. https://doi.org/10.1001/jama.299.13.1561

[30] Stegman, B., Puri, R., Cho, L., Shao, M., Ballantyne, C.M., Barter, P.J., Chapman, M.J., et al. (2014) High-Intensity Statin Therapy Alters the Natural History of Diabetic Coronary Atherosclerosis: Insights from SATURN. Diabetes Care, 37, 3114-3120. https://doi.org/10.2337/dc14-1121

[31] Silverman, M.G., Ference, B.A., Im, K., Wiviott, S.D., Giugliano, R.P., Grundy, S.M., Braunwald, E. and Sabatine, M.S. (2016) Association between Lowering LDL-C and Cardiovascular Risk Reduction among Different Therapeutic Interventions: A Systematic Review and Meta-Analysis. JAMA, 316, 1289-1297.

https://doi.org/10.1001/jama.2016.13985

[32] Schade, D.S., Cavanaugh, B., Ramo, B. and Eaton, R.P. (2016) The Application of the LDL Principle. World Journal of Cardiovascular Diseases, 6, 109-125. https://doi.org/10.4236/wjcd.2016.65012

[33] Zhao, Z., Tuakli-Wosornu, Y., Lagace, T.A., Kinch, L., Grishin, N.V., Horton, J.D., et al. (2006) Molecular Characterization of Loss-of-Function Mutations in PCSK9 and Identification of a Compound Heterozygote. The American Journal of Human Genetics, 79, 514-523. https://doi.org/10.1086/507488

[34] Sabatine, M.S., Giugliano, R.P., Keech, A.C., Honarpour, N., Wiviott, S.D., Murphy, S.A., et al. (2017) Evolocumab and Clinical Outcomes in Patients with Cardiovascular Disease. The New England Journal of Medicine, 376, 1713-1722. https://doi.org/10.1056/NEJMoa1615664

[35] O’Keefe, J.H. (2004) Optimal Low-Density Lipoprotein Is 50 to $70 \mathrm{mg} / \mathrm{dl}$ : Lower Is Better and Physiologically Normal. Journal of the American College of Cardiology, 43, 2142-2146. https://doi.org/10.1016/j.jacc.2004.03.046 
[36] Wilmot, K.A., O’Flaherty, M., Capewell, S., Ford, E.S. and Vaccarino, V. (2015) Coronary Heart Disease Mortality Declines in the United States from 1979 through 2011: Evidence for Stagnation in Young Adults, Especially Women. Circulation, 132, 997-1002. https://doi.org/10.1161/CIRCULATIONAHA.115.015293

[37] Kusters, D.M., Avis, H.J., de Groot, E., et al. (2014) Ten-Year Follow-Up after Initiation of Statin Therapy in Children with Familial Hypercholesterolemia. JAMA, 312, 1055-1057. https://doi.org/10.1001/jama.2014.8892

[38] Mihaylova, B., Emberson, J., Blackwell, L., Keech, A., Simes, J., Barnes, E.H., et al. (2012) The Effects of Lowering LDL Cholesterol with Statin Therapy in People at Low Risk of Vascular Disease: Meta-Analysis of Individual Data from 27 Randomised Trials. The Lancet, 380, 581-590. https://doi.org/10.1016/S0140-6736(12)60367-5

[39] Berry, J.D., Lloyd-Jones, D.M., Garside, D.B. and Greenland, P. (2007) Framingham Risk Score and Prediction of Coronary Heart Disease Death in Young Men. American Heart Journal, 154, 80-86. https://doi.org/10.1016/j.ahj.2007.03.042

[40] Greenland, P., LaBree, L., Azen, S.P., Doherty, T.M. and Detrano, R.C. (2004) Coronary Artery Calcium Score Combined with Framingham Score for Risk Prediction in Asymptomatic Individuals. JAMA, 291, 210-215.

https://doi.org/10.1001/jama.291.2.210

[41] Nasir, K., Rubin, J., Blaha, M.J., Shaw, L.J., Blankstein, R., Rivera, J.J., Khan, A.N., et al. (2012) Interplay of Coronary Artery Calcification and Traditional Risk Factors for the Prediction of All-Cause Mortality in Asymptomatic Individuals. Circulation: Cardiovascular Imaging, 5, 467-473. https://doi.org/10.1161/CIRCIMAGING.111.964528

[42] Ross, R. (1999) Atherosclerosis-An Inflammatory Disease. The New England Journal of Medicine, 340, 115-126.

[43] Catapano, A.L., Graham, I., De Backer, G., et al. (2016) ESC/EAS Guidelines for the Management of Dyslipidemias. European Heart Journal, 37, 2999-3058. https://doi.org/10.1093/eurheartj/ehw272

[44] Hayward, R.S.A. (1997) Clinical Practice Guidelines on Trial. CMAJ, 156, 1725-1727.

[45] Pagidipati, N.J., Navar, A.M., Mulder, H., Sniderman, A.D., Peterson, E.D. and Pencina, M.J. (2017) Comparison of Recommended Eligibility for Primary Prevention Statin Therapy Based on the US Preventive Services Task Force Recommendations vs. the ACC/AHA Guidelines. JAMA, 317, 1563-1567. https://doi.org/10.1001/jama.2017.3416

[46] Pencina, M.J., Navar-Boggan, A.M., D’Agostino, R.B., Williams, K., Neely, B., Sniderman, A.D. and Peterson, E.D. (2014) Application of New Cholesterol Guidelines to a Population-Based Sample. The New England Journal of Medicine, 370, 1422-1431. https://doi.org/10.1056/NEJMoa1315665

[47] Lazar, L.D., Pletcher, M.J., Coxson, P.G., Bibbins-Domingo, K. and Goldman, L. (2011) Cost-Effectiveness of Statin Therapy for Primary Prevention in a Low-Cost Statin Era. Circulation, 124, 146-153. https://doi.org/10.1161/CIRCULATIONAHA.110.986349

[48] AHA Committee; Stroke Statistics Subcommittee (2016) Heart Disease and Stroke Statistics-2016 Update: A Report from the American Heart Association. Circulation, 133, e38-e360. https://doi.org/10.1161/CIR.0000000000000350

[49] Cabana, M.D., Rand, C.S., Powe, N.R., Wu, A.W., Wilson, M.H., Abboud, P.-A.C., et al. (1999) Why Don't Physicians Follow Clinical Practice Guidelines? A Frame- 
work for Improvement. JAMA, 282, 1458-1465.

https://doi.org/10.1001/jama.282.15.1458

[50] Bothwell, L.E., Greene, J.A., Podolsky, S.H. and Jones, D.S. (2016) Assessing the Gold Standard-Lessons from the History of RCTs. The New England Journal of Medicine, 374, 2175-2181. https://doi.org/10.1056/NEJMms1604593 